\title{
Principal component analysis for human gait recognition system
}

\author{
Othman O. Khalifa, Bilal Jawed, Sharif Shah Newaj Bhuiyn \\ Department of Electrical and Computer Engineering, International Islamic University Malaysia, Malaysia
}

\begin{tabular}{l} 
Article Info \\
\hline Article history: \\
Received Jan 7, 2019 \\
Revised Feb 25, 2019 \\
Accepted Mar 30, 2019 \\
\hline Keywords: \\
Biometric \\
Gait recognition \\
Human identification \\
PCA \\
Silhouette \\
Video surveillance \\
\hline
\end{tabular}

\begin{abstract}
This paper represents a method for Human Recognition system using Principal Component Analysis. Human Gait recognition works on the gait of walking subjects to identify people without them knowing or without their permission. The initial step in this kind of system is to generate silhouette frames of walking human. A number of features couldb be exytacted from these frames such as centriod ratio, heifht, width and orientation. The Principal Component Analysis (PCA) is used for the extracted features to condense the information and produces the main components that can represent the gait sequences for each waiking human. In the testing phase, the generated gait sequences are recognized by using a minimum distance classifier based on eluclidean distance matched with the one that already exist in the database used to identify walking subject.
\end{abstract}

Copyright () 2019 Institute of Advanced Engineering and Science. All rights reserved.

Corresponding Author:

Othman O. Khalifa,

Department of Electrical and Computer Engineering,

International Islamic University Malaysia,

Jalan Gombak, 53100 Kuala Lumpur, Malaysia.

Email: khalifa@iium.edu.my

\section{INTRODUCTION}

The world security has always been at risk places like airports, train stations, shopping malls, national borders, public parks, national monuments and other public areas have always been at risk despite having several security measures. These places use several years old identification method which cannot be used now when the security level requires high technology which is unobtrusive. If considering the case of tube station which is very busy, use of old identification methods for verification is obviously infeasible. Therefore, for these issues the investment and development of biometric identification methods (eye, fingers, hand geometry and human gait recognition system) have attracted the attention of the government and leading development companies. Gait recognition system is a modern technology which does not require permission of the targets, it works on unobtrusive technology that detects by human gait gesture if a person is a thread to security or if he is behaving suspicious $[1,2]$.

There are several biometric identifications but if those are compared to human gait/gesture identification/authentication, it has some exclusive characteristics. Gait is unobtrusive i.e. it works in such a way that it does not require permission of the observing subject and can work from a distance, whereas other biometric needs a physical response from the subject such as fingers, eyes, face and voice etc. Gait has several disadvantages as well at this platform of being an undeveloped field till now, there is a limitation to gait technology when it comes to human body for identification. Gait technology works on a basis of a video captured in busy or customized places but even after this its result cannot be accurate enough to take decisions. Gait is unique distinguishable biometric feature as each person has a distinctive walking style which is easily understood from biomechanical viewpoint. Therefore, by several researches done on gait it is concluded that human gait identification depends on clothes, the place of walking, stress and can change with different emotion feelings [3-6]. Thus, it is hard to deploy such an identification method and this is what that discriminates gait from other biometric identification methods. Gait when combined with other biometric 
identification method can be of great use in several identification applications due to its uniqueness. Figure 1 shows the human gait steps analysis. Therefore, gait is not used as the only identification method but some field which is unique and full potential in multi-biometric applications.

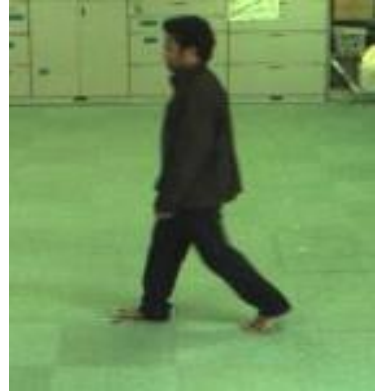

(a)

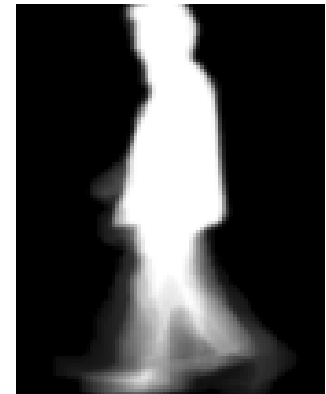

(b)

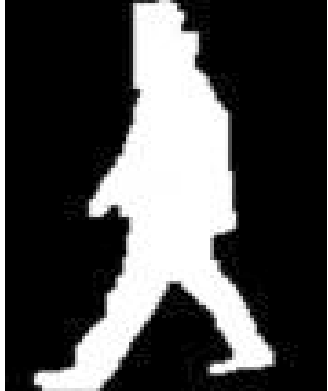

(c)

Figure 1. Human gait analysis, (a) Human gait--Silhouette, (b) Gait feature, (c) Size--normalized silhouette

This paper presents human gait recognition system. In this paper, we are only focusing on human tracking using background subtraction, gait feature extraction and recognition using Principle Component Analysis (PCA). The rest of this paper is organized as follows. Section 2 presents the definition of human gait recognition, background subtraction \& PCA, Section 3 presents the related works, Section 4 presents the details of the proposed method, the Section 5 the experimental results and the last is conclusion.

\section{A GENERAL GAIT RECOGNITION SYSTEM}

Human Gait Recognition is a type of bio-metric identification system that identifies subjects based on their body posture known as 'Human Gaits'. The human gait is unique to every individual and therefore, a person can be detected by their gait. Gait recognition system is a modern technology which does not require permission of the targets, it works on unobtrusive technology that detects by human gait gesture if a person is a thread to security or if he is behaving suspicious. Figure 2 shows human gait recognition process.

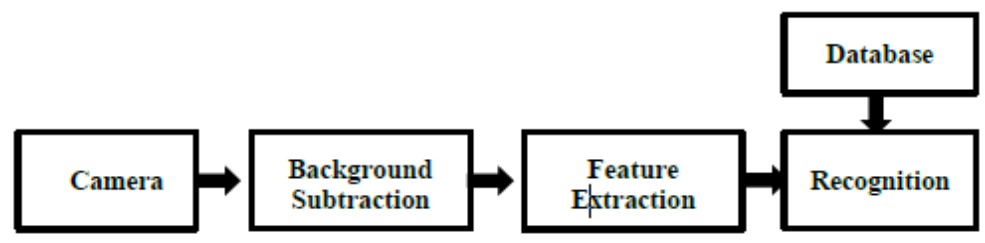

Figure 2. Human gait recognition process

Current approaches to gait recognition can be divided into two categories: Appearance-based ones $[4,5]$ that deal directly with image statistics and Model-based ones [6-8] that first model the image data and then analyze the variation of its parameters. The majority of current approaches are the appearance-based approaches which are simple and fast. But the silhouette appearance information is only indirectly linked to gait dynamics. Figure 3 shows block diagram of the gait recognition system.

For Human Gait recognition process, background subtraction is very important step. If the system can't separate the human from the main background then, the whole system will fail to match or might fall into the false match category [8]. To decrease the false acceptance rate (FAR), we need to use the proper background subtraction method with noise filtering. Figure 4 shows background removal process flow. 


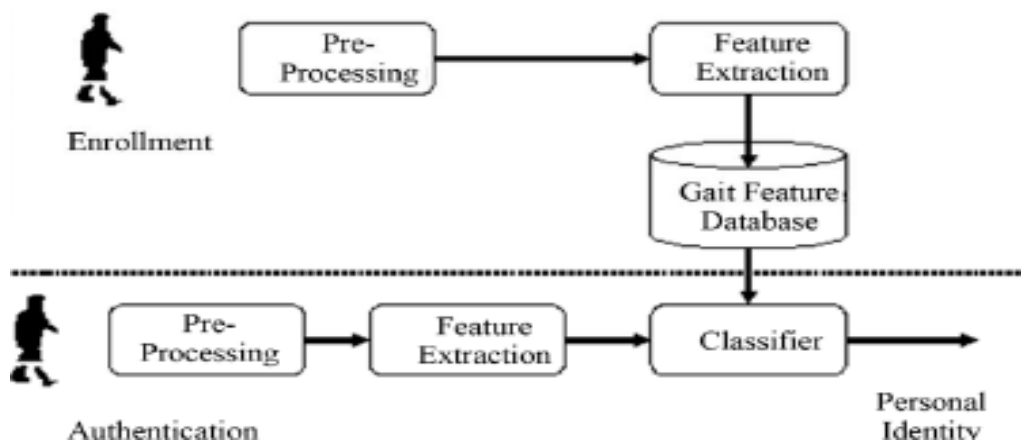

Figure 3. Block diagram of the gait recognition system

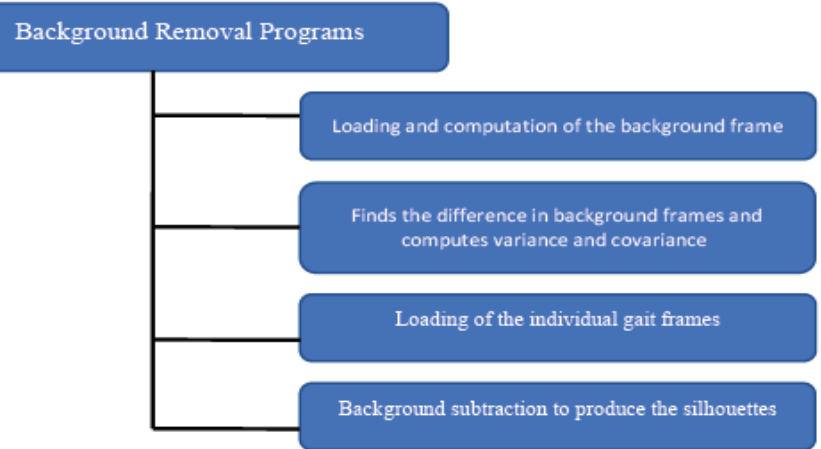

Figure 4. Background removal process flow

After segmenting the Human from the original background, we need to create the database of normalized feature from Silhouette images. Below is one of our silhouette image after background subtraction process. Our process removed background very perfectly for tracking human. Figure 5 shows sample of walking gait video.

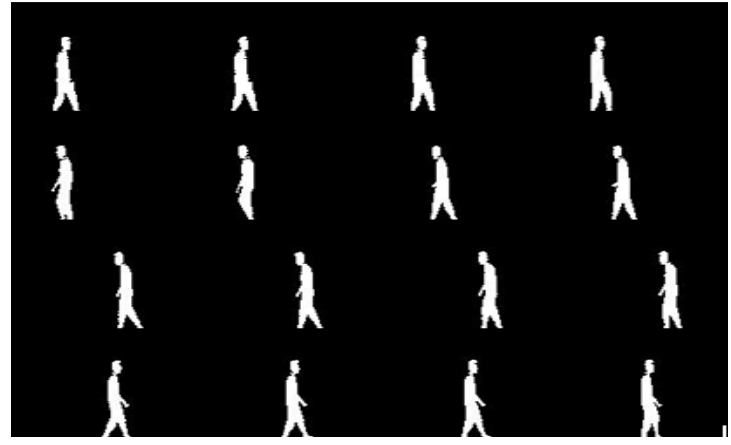

Figure 5. Sample of walking gait video

For gait recognition or matching, we can't use template based matching. Because of scaling, rotating and skewing of image. These appearances will happen because of multiple viewpoints or camera sensors, illumination or lighting changes and the human itself for facial expression. To handle this issues, we need to increase the number of templates or we can represent the problem as multi-dimensional data of a single image or point. To solve the higher problem efficiently, we need to apply Principle Component Analysis (PCA) algorithm. Below we can see how PCA can be formulate for $\mathrm{N}$ number of images into a matrix form to calculate eigen-space. Eigen-vectors span eigen-space like $E_{1}, E_{2}, E_{3}, E_{4} \ldots E_{n}$. Figure 6 shows PCA representation of $\mathrm{N}$ number of images in matrix form. 


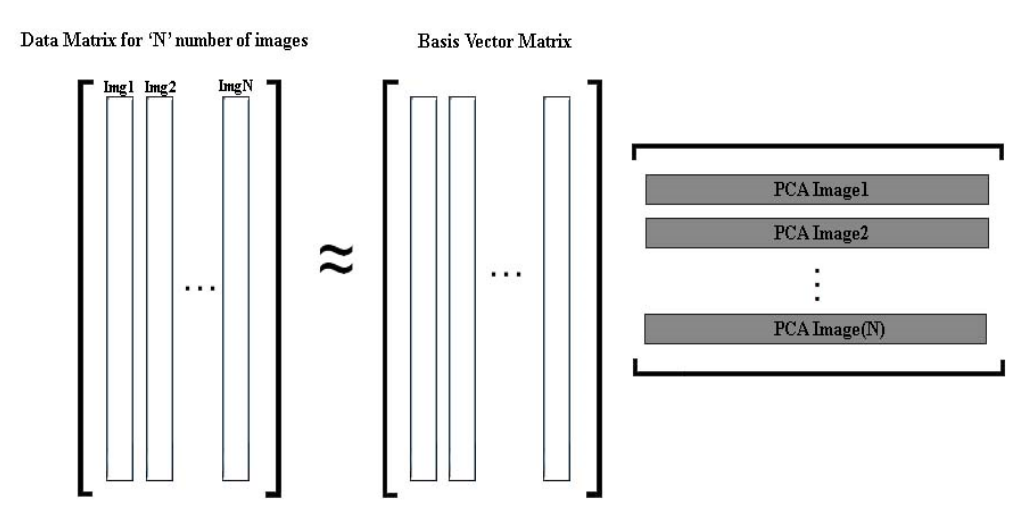

Figure 6. PCA representation of $\mathrm{N}$ number of images in matrix form

\section{GAIT FEATURE REPRESENTATION}

Gait recognition representation is an important step in gait biometric recognition. It represents content of the individual image and the pattern of human walking simultaneously. It can be divided into:

a. Appearance-Based Methods: they are widely used in gait representation. It is based on analysing human motion and extracting distinct features. The recognition process in this approach is based on subject detection, silhouette extraction, and feature extraction and classification. After detecting the human motion captured by the sensor (camera), background subtraction is performed on the detected human gait, which is followed by feature extraction on the detected gait features. After feature extraction, dimensionality reduction is performed because the extracted features have high dimensionality information. Finally, a classification technique is performed on the reduced dimension gait features.

b. Model-Based Methods: they are represent a gait with body segments, joint positions, or pose parameters. They have a cluster of static or dynamic body parameters through modelling or tracking body components likes limbs, legs, arms and thighs. From these model parameters are then produced gait signatures used for identification and recognition of an individual. Model-based approaches proven that it is view-invariant and scaleindependent. These advantages are significant for practical applications, because it is unlikely that reference sequences and test sequences are taken from the same viewpoint [6]. Besides that, model-based approach are very sensitive to quality standard of image of gait sequences. Thus, high quality images of gait sequences are needed to get high accuracy result.

\section{RELATED WORKS}

Gait recognition is non intrusive operates at a distance without subject cooperation. Individual biometrical identification system is achieved with methods such as fingerprints, face, hand geometry, iris, voice, signature, and gait [3], but all of them, excluding gait, needs to be captured only by physical contact or at a close distance from the recording sensor [4]. Many researchers have studied the idea of an individual's gait being unique, particularly material that provides several different approaches to achieve a successful working gait recognition system. We systematically analyzed the stages involved in a human gait recognition system and the components required in the process

Model based approaches have a model in which the gait sequence is recorded under favorable parameters. The advantage of model approach over holistic approach is that model approach is view and scale invariant as it is very difficult to capture the image every time from the same view point as stored in the database for reference. There are specific parameters that are fed in the database, therefore in this approach only high quality selected gaits are chosen from the gait sequence. There is a lot of noise and other disturbance such as self-blockage is noticed which can destroy or disturb the parameters needed for gait sequence. Therefore, a different technique can be used in which a multi camera gait learning system should be used to obtain a better gait sequence. A similar process was used in [5] using a multi-view human gait learning method which depends on the retrieved static gait criterion that are the dimensions acquired from static gait sequence. Gait dynamics are not involved. The static specifications taken in [8-10] are the maximum difference between abdomen and feet, the length (height), the difference between head and abdomen and the difference between the feet. The static framework is not effected by the view i.e. its view invariant, which makes it most preferable for recognition feature [11, 12]. 
Another approach which was motion based method. It used optical flow to detect moving objects and extract gait sequences [13-15]. Table 1 shows a summary of the most common approaches for Human Gait Recognition.

Table 1. Summary of the most common approaches for gait recognition

\begin{tabular}{lll}
\hline \multicolumn{1}{c}{ Author(s), Year(s) } & \multicolumn{1}{c}{ Approach } & \multicolumn{1}{c}{ Limitations } \\
\hline $\begin{array}{l}\text { Nikolaos V. Boulgouris, Dimitrios } \\
(2005)\end{array}$ & $\begin{array}{l}\text { Gait Recognition: A challenging signal } \\
\text { processing technology for biometric } \\
\text { identification", }\end{array}$ & $\begin{array}{l}\text { Recognition rate } 58 \%, \\
\text { Motion of hip is fixed. }\end{array}$ \\
$\begin{array}{ll}\text { L. Wang, M.S. Nixon (2003) } \\
\text { Using modified ICA }\end{array}$ & $\begin{array}{l}\text { Walking only in } 0^{\circ}, 45^{\circ}, 90^{\circ} \text { to the fixed } \\
\text { camera. } \\
\text { L. Huang. C. Harris, M. Nixon (1999) }\end{array}$ & $\begin{array}{l}\text { Using Temporal Templates } \\
\text { video }\end{array}$ \\
$\begin{array}{l}\text { Faustini Libent Ishabailu, Dr. Pei Zhijun, } \\
\text { Abdalla Mohamed Hambal (2014) }\end{array}$ & $\begin{array}{l}\text { Recognition System Based On Principal } \\
\text { Component Analysis (PCA) }\end{array}$ & $\begin{array}{l}\text { Walking only in } 0^{\circ}, 45^{\circ}, 90^{\circ} \text { to the fixed } \\
\text { camera on a tri-stand }\end{array}$ \\
\hline
\end{tabular}

\section{PROPOSED METHOD}

The proposed approach is separating a moving person through a video from the background scene. Therefore, the collection of date is the first step. Then the method of background removal and reduce the noise to a certain level. The segmentation and tracking are the next stage. This phase is called Human Detection. The methodology has been shown in Figure 7.

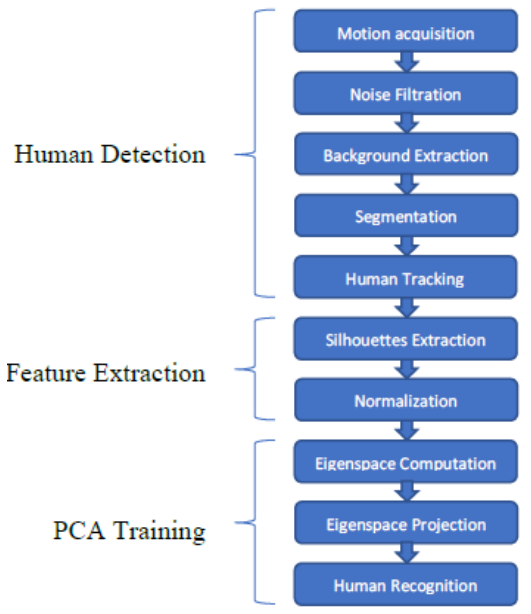

Figure 7. Flowchart of methodology

In human detection phase, a typical video stream has several frames of background only, followed by many frames of a person walking across the scene, followed by several more frames of background only. An example may be ten frames of only background, 130 frames of the person walking, followed by fifteen frames of background. After the video is converted to jpeg files, the first task is to identify those frames in the beginning and at the end which contain only background. These files are identified in program which averages the red, green, and blue (RGB) intensities of each individual pixel across all these frames. For instance, if there are a total of twenty-five frames of only background. The RGB intensity of pixel 1, 1 in each of these twenty-five frames is averaged. This is done for each of the 345,600 pixels (image size 480x720). These average background pixels are then combined into a new image that represents the average background scene. The program also displays a subplot of the RGB intensities.

Then subtracts the average background frame from each of the background frames and produces a scatter plot of a select number of the differences. The reduced frames are used to compute the variance and covariance in the RGB intensities which will be used to determine whether the pixel is solely background or person. At the end, compares the RGB intensity of each pixel to the average background intensity of the same pixel. Those pixels within the covariance region are deleted and those pixels outside the covariance region are assumed to be the individual. Thus, the background of the picture has been removed from the person. 


\section{EXPERIMENTAL RESULTS}

\subsection{Background subtraction}

The data was taken offline from database and background subtraction was performed. Each file is presented as a frame of size $480 \times 720$ (480 rows of pixels by 720 columns of pixels and each pixel has three RGB dimensions). That is there are 1,036,800 pieces of information for each frame or a total of $181,440,000$ pieces of information for a single individual video. Next, we subtracted the average background frame from each of the background frames which produced a scatterplot of a select number of the differences. The reduced frames are used to compute the variance and covariance in the RGB intensities. With this said the RGB variance in the first set of background data is 8.0781, 7.2496 and 8.5232, respectively. Figure 8 shows average background.

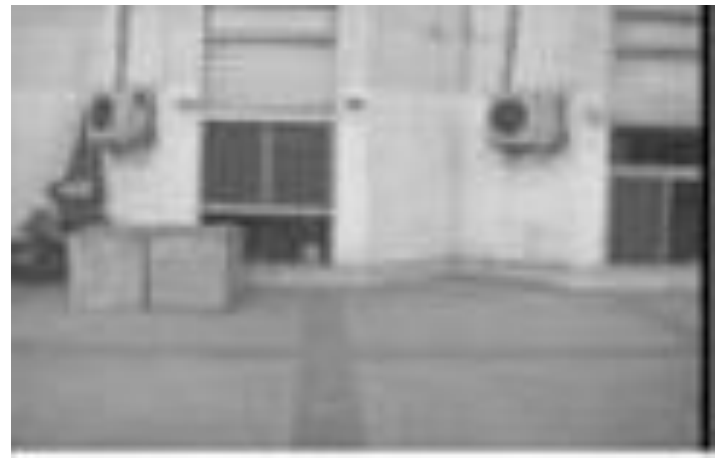

Figure 8. Average background

\subsection{Silhouettes extraction}

After testing every pixel in every frame and assigning a one or zero to each pixel in the picture, a silhouette of the image was made by coloring the zero pixels black and the one pixels white. Thus a series of images was created with a black background and white silhouette image. As in the image frames of the individual walking across the screen, this series of silhouette frames is put into an avi file in the MATLAB program in order to show motion in the figure

\subsection{Noise filtration}

The default of the medfilt 2 filter performs a median filtering on the data in a three-by-three neighborhood with the pixel in question being the center. Figure 9 shows noise filtration (a) unfiltered image frame (b) filtered image frame.

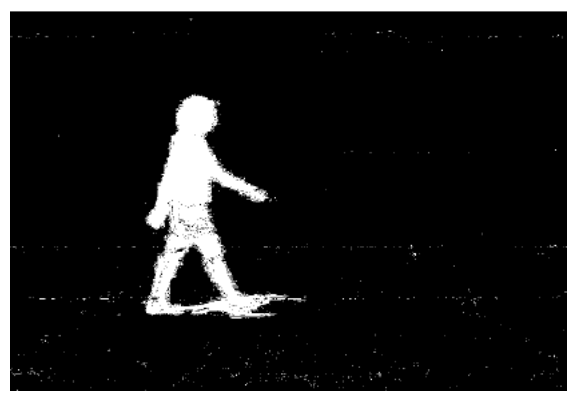

(a)

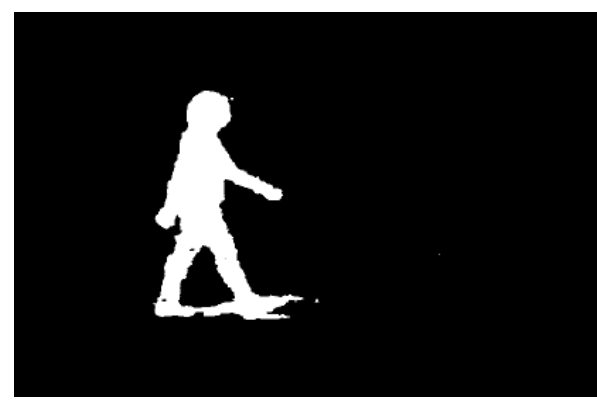

(b)

Figure 9. Noise filtration, (a) UnFiltered image frame, (b) Filtered image frame

The silhouettes obtained where stored in the database of MATLAB. This subsection contains the results of few the trained samples from the database we employed PCA training and the results after the simulation by using MATLAB software are depicted in Table 2.

Bulletin of Electr Eng and Inf, Vol. 8, No. 2, June 2019: $569-576$ 
Table 2. PCA gait recognition results

\begin{tabular}{ccccc}
\hline Trail & Dataset & Match ID & Time $(\mathrm{sec})$ & Feedback \\
\hline 1 & $00 \_1$ & 1 & 26.2541 & Positive Match \\
2 & $00 \_2$ & 2 & 0.036601 & Positive Match \\
3 & $00 \_3$ & 3 & 30.505 & Positive Match \\
4 & $00 \_4$ & 4 & 35.104 & Positive Match \\
5 & $45 \_1$ & 5 & 41.5777 & False Match \\
6 & $45 \_2$ & 6 & 54.8304 & False Match \\
\hline
\end{tabular}

\section{CONCLUSIONS}

Human Gait is a behavioural biometric trait that can be used as an effective biometric feature for human identification. It gained attention of the researches recently. Biometrics such as fingerprints, retina, palm and voice needs physical permission of the subject whereas gait technology needs no authorization from the subject as it works from distance on human postures. A model free-based human identification method using the gait recognition algorithm is proposed in this paper. The efficiency of the proposed algorithm for human identification is tested using the national Gait dataset. to which PCA training is applied. The noise filtration is applied to the silhouettes to get a better picture quality. For future, such a human gait recognition should be developed which can work in all kind of surrounding including places like metro stations and banks. The database also needs to be advanced by having larger database with more sequences and subjects. The algorithm applied above is very complicated, a better formula should be developed to ease the process of gait recognition. The camera should be used multi angle which can record a video of a walking subject from different angle at the same time, this will also help in making the algorithms easier

\section{REFERENCES}

[1] N. V. Boulgouris, D. Hatzinakos and K. N. Plataniotis, "Gait recognition: a challenging signal processing technology for biometric identification," in IEEE Signal Processing Magazine, vol. 22, no. 6, pp. 78-90, Nov. 2005.

[2] B. Jawed, O. O. Khalifa and S. S. Newaj Bhuiyan, "Human Gait Recognition System," 2018 7th International Conference on Computer and Communication Engineering (ICCCE), Kuala Lumpur, 2018, pp. 89-92.

[3] J. Little and J. Boyd, "Recognizing people by their gait: The shape of motion", Videre, Int. J. Computer Vison, 1998.

[4] L. Lee and W. E. L. Grimson, "Gait analysis for recognition and classification," Proceedings of Fifth IEEE International Conference on Automatic Face Gesture Recognition, Washington, DC, USA, 2002, pp. 155-162.

[5] Agung Nugroho Jati, Astri Novianty, Nanda Septiana, Leni Widia Nasution, "Comparison Analysis of Gait Classification for Human Motion IdentificationUsing Embedded Computer", International Journal of Electrical and Computer Engineering (IJECE), December 2018, 8(6): 5014 5020.

[6] P. S. Huang, C. J. Harris and M. S. Nixon, "Human gait recognition in canonical space using temporal templates," in IEE Proceedings - Vision, Image and Signal Processing, vol. 146, no. 2, pp. 93-100, April 1999.

[7] A. Johnson and A. Bobick, "A multi view method for gait recognition using static body parameters", 3rd Int. Conf. Audio and video based biometric person authentication, Halmstad, Sweden, June 2001.

[8] D. Cunado, M.S. Nixon, and J.N. Carter, "Automatic extraction and description of human gait models for recognition purposes”, Comput. Vis. Image Understand, vol. 90, pp. 1-14, 2003.

[9] A. Johnson and A. Bobick, "A multi view method for gait recognition using static body parameters, "in 3rd Int. Conf. Audio and video based biometric person authentication, Halmstad, Sweden, June 2001.

[10] Y. Yang and M. Levine, "The Background Primal Sketch: An approach for Tracking Moving Objects," Machine Vision and Applications, 1992.

[11] Faustini Libent Ishabailu, Pei Zhijun, Abdalla Mohamed Hambal, "Human Gait Recognition System Based On Principal Component Analysis", International Journal of Science and Research, April 2017 , Volume 6 Issue 4, pp. 989 - 994.

[12] N.A. Makhdoomi, T.S. Gunawan, M. Kartiwi, "Evaluating the effect of viewing angle in different conditions for gait recognition," ARPN Journal of Engineering and Applied Sciences, 2017, 12 (16). pp. 4729-4735.

[13] S.D. Mowbray and M.S. Nixon," Automatic gait recognition via Fourier descriptors of deformable objects," in Proc. Int. Conf. on Audio and video based Biometric Person Authentication, Guikford, UK, 2003.

[14] D. K. Wagg and M. S. Nixon, "On automated model-based extraction and analysis of gait," Sixth IEEE International Conference on Automatic Face and Gesture Recognition, 2004. Proceedings., Seoul, South Korea, 2004, pp. 11-16.

[15] Y. Li, L. Yan, H. Qian, "A Gait Recognition System using GA-based C-SVC and Plantar Pressure," Indonesian Journal of Electrical Engineering and Computer Science (IJEECS), 11(10), pp. 6135-6142, 2013. 


\section{BIOGRAPHIES OF AUTHORS}

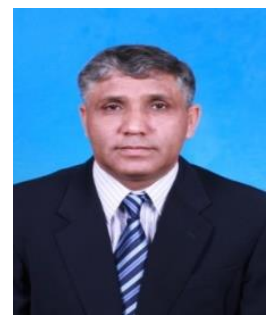

Othman Omran Khalifa received his Bachelor's degree in Electrical Engineering from the Garyounis University, Libya in 1986. Prof. Khalifa obtained his MSc and PhD in 1996, 2000 respectively from Newcastle University, UK. He joined International Islamic University Malaysia in August 2002. Prof. Khalifa served as a head of department of Electrical and computer Engineering, IIUM from July 2005 until December 2014. Prof. Khalifa is Charter Engineer and a senior member of IEEE, USA and a member IET, UK, a member of the International Association of Engineers (IAENG). Prof. khalifa was the chairman of the International Conference on Computer and Communication Engineering (ICCCE), 2006, 2010, 2012, 2014. Prof. Khalifa has extensively contributed through his writings in international journals, conferences and books. He published more than 410 publications including 10 books. $\mathrm{He}$ is a member of many international advisory boards for many international conferences.

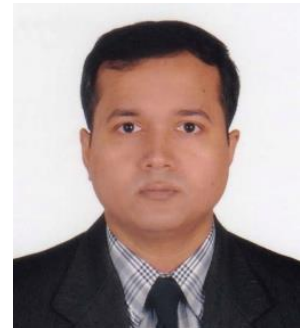

Sharif Shah Newaj Bhuiyan obtained his BSc degree in Computer Science and Engineering from Ahsanullah University of Science and Technology (AUST), Bangladesh in 2008. Currently he is completing his MSc in Computer and Information Engineering from IIUM, Malaysia. He has $10+$ years of professional experience in Software Engineering. His area of interests includes to Image Processing, Deep Learning, Graph Theory, Distributed Computing and Cloud Computing. 